\title{
CAD Based Electric Transporter Path Planning and Production Storage Optimization Using Genetic Algorithm - Industrial Case Study
}

\author{
Miha Kovačič, Goran Đukić, Brigita Gajšek, Klemen Stopar
}

\begin{abstract}
Steel Ltd. is one of the largest flat spring steel producers in Europe. There are two production lines after rolling - one for flat bars and the other for round bars. The flat bars production generally consists of visual examination, straightening and cutting operation. In addition, heat treatment or magnetic particle testing could be conducted. On the other hand, the round bars production consists generally of straightening, automatic control line control, chamfering and cutting. In addition, heat treatment is possible. For manipulation of the material in the rolling plant, the electric transporter and several cassettes are used. In the paper path planning and production storage optimization (i.e. storage spaces for cassettes) were conducted using genetic algorithm. The production storage is actually the space between main transport passage and individual operations. In the research the universal system using CAD geometry is presented where AutoCAD environment and in-house developed AutoLISP system were used. The production storage storage spaces for cassettes (location and orientation) with corresponding electric transporter trajectories are represented as CAD objects and thus form individual solution/organism. The organisms undergo simulated evolution. The results of the evolution are compared with actual production storage in the steel plant.
\end{abstract}

Keywords: genetic algorithm; optimization; path planning; production storage; steel industry

\section{INTRODUCTION}

Production space is a space primarily intended for production. Any stock of material or products in production is a loss because it binds capital, occupies production's space and requires additional manipulation. Especially products in the production hall are not desirable at all. Nevertheless, intermediate storage places for products in production are a reality. They serve as a buffer for times when the outbound warehouse is out of service or its resources are busy with shipping.

Research on intermediate storage spaces is common in the scientific literature. The buffer allocation problem concerning the size and location of storage between the stages of a flow line is a critical research area in the design of production lines. For example, Nori Prakasa [1] introduced an analytical study of several aspects of two-stage production systems with variable operation times and provision for intermediate storage. The problem of balancing the production system is discussed at some length. It is shown that the production rate improves on allotting a slightly higher load to the less variable stage. Martin [2] extends results of previous models for optimizing interstation buffer capacities for short production lines. His paper refines and extends previous results for lengthier systems and for generally-distributed workstation. His results are presented in a table that provides the line designer with the essential coefficients to find the optimal buffer capacities for many realistic systems. Mak [3] developed a simple optimal buffer capacity model for series production line where the service time variability is described by the normal distribution and the buffers are allocated the same capacity. An expression is derived for the buffer capacity, which minimizes the sum of the delay, inventory carrying, and storage facility costs.

We could continue to summarize the problems discussed in the field of intermediate storage areas in production hall, but most of the scientific work relates to inventories of materials and semi-finished products in production.
Discussion about storage of finished products in production hall is not common. Materials and semi-products are mostly stored in crates and standard pallets, which allows the use of agile, standard vehicles like forklifts that do not require much room for manoeuvre when handling transport units. In our case, we are dealing with cassettes measuring $3 \times 7 \mathrm{~m}$.

In our case, as a difference with respect to frequent research direction observed in scientific literature, the locations and areas of intermediate storage area are defined. Cassettes are deposited on the ground within the physically limited storage area. Given the intended flow of finished products from production to the warehouse, we are looking for the most appropriate way of stacking cassettes within the space available for intermediate storage for products in production hall. We are slightly constrained by the technical features of the electric transporter with which the cassettes are carried. Because of the specific nature of electric transporter's driving in turns, it is not possible to deposit cassettes completely arbitrarily. To solve the problem of cassette distribution across storage area, we expect answers from scientific paper authors in the field known as the facility layout problem. Layout problems generally differ on layout type, presentation (i.e. shape of facility), constrains (i.e. problem formulation) and also on solving approach [4-6]. Layout presentation is certainly one of the most important features. It can be numerical or geometrical including numerical and/or geometrical constrains [4-6]. Several approaches can be used for its solving including artificial intelligence methods for example taboo search [7], fuzzy logic [8], ant colony [9], particle swarm optimization [10] or genetic algorithms [11]. Despite the variety of methods used in mentioned scientific papers, we did not find a paper that would directly answer our question about the optimal arrangement of cassettes across the available intermediate storage area for finished products, taking into account the limitations of the electric transporter due to its limitations about driving in turns. 


\section{METHODOLOGY}

For solving the problem computer aided design (CAD) and genetic algorithm were used.

The survey regarding CAD geometrical representation of the layout problems reviles several implementations of $C A D$ software for solving problems at assembly [12], path planning $[13,14]$ to production facility rearrangement $[15$, $16]$.

In the paper [17] the CAD system for path planning of extruder was used. Deposition path is constructed from several surfaces using different strategies and can be finally connected into continuous path. In this way the efficiency of extruder can be enhanced.

The article [12] explores the usage of CAD systems for digital data acquisition, motion capture, rendering, virtual assembly, assembly planning, prototyping, training, evaluation and testing of assembly systems.

The authors in [13] used CAD for generation and examination of several robot movement scenarios taking into account ergonomics, quality and productivity. The system was implemented into automotive industry test case.

In the paper [18] CAD system was used for generation of robot trajectories which can be used for off-line automatic motion planning and simulation of robot movements taking into account kinematics and dynamic performances.

The research co-written by the first author of this paper [11] discusses using genetic algorithms for optimal rearranging of the machines in the finishing plant after the available space was drastically reduced due to installation of a new continuous rolling mill line. AutoCAD and in-house developed AutoLISP genetic algorithm based system was used for layout optimization. The machines can be represented as two-dimensional regions or three-dimensional solids without any kind of geometrical restrictions.

Similar article [19] presents CAD based universal solution for cutting stock problem. The parts which are to be cut from stock-material with minimal loss and also the stockmaterial itself are presented as CAD geometries without any kind of geometrical constraints. In the paper also AutoCAD, AutoLISP and genetic algorithm was used as search strategy.

In this paper, the universal system using CAD geometry is presented. AutoCAD environment and in-house developed AutoLISP system were used. The developed system was used for path planning and production storage optimization (i.e. storage spaces for cassettes) in steel plant in Slovenia. Genetic algorithm was also used. The intermediate storage spaces in production (storage spaces for cassettes (location and orientation)) with corresponding electric transporter trajectories are represented as CAD objects and thus form individual solution/organism.

On the end of this paper, the developed system is presented. Afterwards, industrial case of path planning and production storage optimization is shown. The results of the optimization are compared with the actual production storage space. The paper ends with conclusions and guidelines for future work are given.

\section{INDUSTRIAL CASE STUDY}

Štore Steel Ltd. is one of the largest flat spring steel producers in Europe. There are two production lines after rolling - one for flat bars and the other for round bars. The flat bars production generally consists of visual examination, straightening and cutting operation. In addition, heat treatment or magnetic particle testing could be conducted. On the other hand, the round bars production consists generally of straightening, automatic control line control, chamfering and cutting. In addition, heat treatment is possible.

\section{THE ARCHITECTURE OF PATH PLANNING AND PRODUCTION STORAGE OPTIMIZATION SYSTEM}

For manipulation of products in the rolling plant the electric transporter and several detachable cassettes $(3 \times 7 \mathrm{~m})$ are used (Fig. 1). The electric transporter is first driven below the cassette located along the production line and after it lifts the cassette up from the ground. Once loaded, it enters the main transport path and through it to one of the intermediate storage areas in production. The material is stored in three storage areas in production hall. Fig. 2 shows the location of main transport path and three storage areas (coloured grey) in AutoCAD.

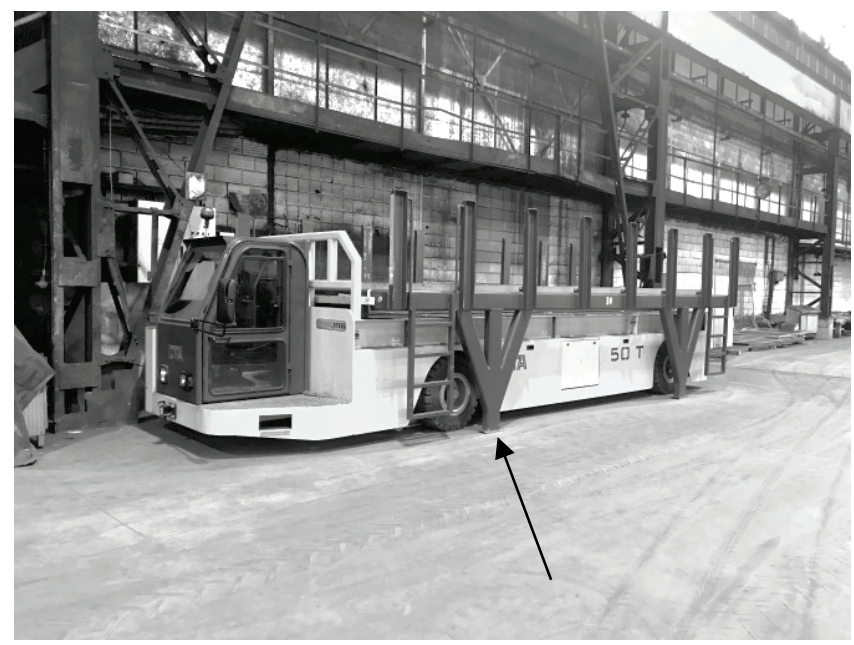

Figure 1 The electric transporter with blue cassette [1]

The proposed approach is created within the AutoCAD system. The AutoLISP/VisualLISP is used as the programming language. The LISP language is incorporated into all contemporary AutoCAD systems. The AutoLISP allows the user to access the AutoCAD's internal drawing database and to manipulate graphical entities. The developed CAD system consists of following modules:

- geometry definition,

- constraint definition,

- evaluation and

- repetitive changing and evolution using different evolutionary operators (e.g. crossover, mutation).

Each cassette represents a part of the storage layout (Fig. $3)$. It has its location, orientation and individual paths 
consisting of turns and straight lines leading from/to main transport path. According to technical specification of transportation vehicle, in our case electric transporter, turns can be constructed in a way that is actually feasible. Simplified construction of turns was used in the research.

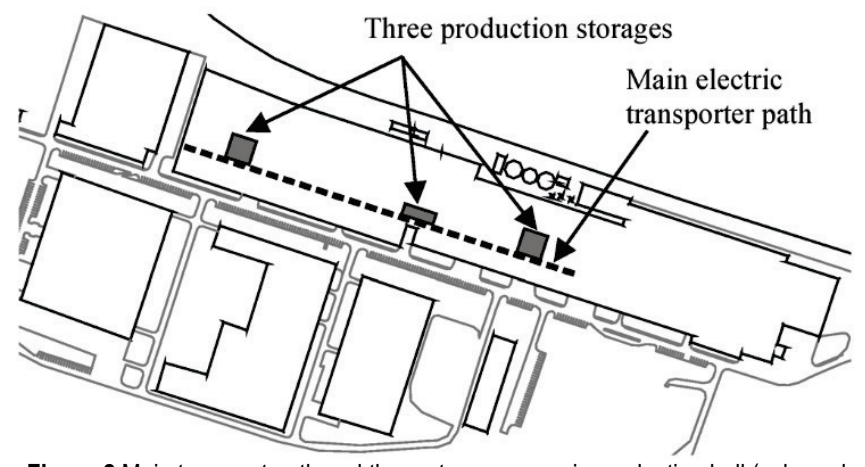

Figure 2 Main transport path and three storage areas in production hall (coloured grey) in AutoCAD

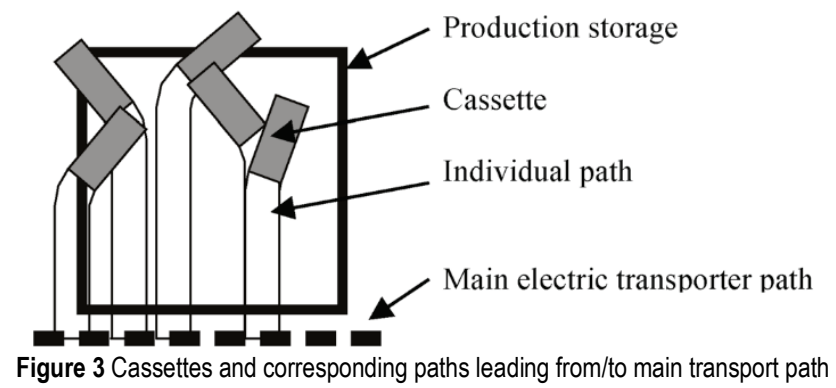

Regarding constrains it is needed to emphasize that the cassettes should be positioned into the storage area, the orientation can be arbitrary or not and also the minimal turning radius is set according to technical specifications of the electric transporter. The number of the cassettes is arbitrary.

The storage layout is evaluated based on:

- $\quad$ area of all cassettes inside storage area $(A)$,

- intersection area between cassettes $(A c)$,

- area of cassettes outside storage area (Acos) and

- area of electric transporter's paths outside storage area (Aetos).

Intersection area between cassettes

Area of cassettes outside production storage

Area of electric transporter paths outside production storage

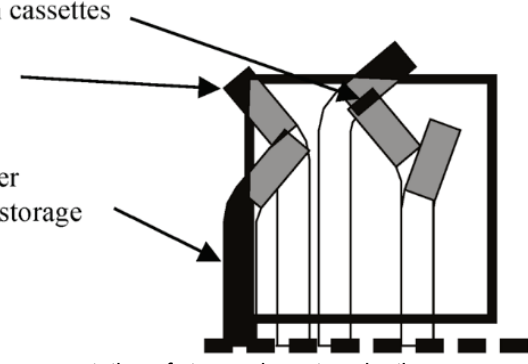

Figure 4 Graphical representation of storage layout evaluation

The evaluation function $F$ is defined as:

$F=w_{A} \cdot A-w_{A c} \cdot A c-w_{A \cos } \cdot A \cos -w_{\text {Aetos }} \cdot$ Aetos where $w_{A}, w_{A c}, w_{A c o s}$ and $w_{A e t o s}$ are corresponding weights, which can be adjusted. In the paper, following weights were used:

$F=1 \cdot A-10 \cdot A c-10 \cdot A \cos -10 \cdot A e t o s$

For the purpose of this research, the genetic algorithm was used as a search strategy. The genetic algorithms are search heuristics, which simulate the natural evolution of living beings [18]. The genetic algorithms have been already used by the authors of scientific papers for solving various problems [21-25].

The organisms (i.e. production storage layouts) consist of genes (i.e. individual electric transporter's paths). Each gene contains the data on location and orientation of the cassette. Based on each cassette location (i.e. reference point) and orientation the cassette, straight path and turn are automatically generated in the AutoCAD (Fig. 5). The best organism in the population represents the best production storage layout.

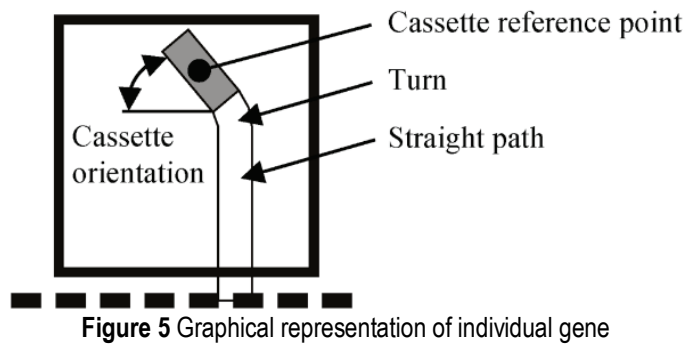

For changing organisms, the following genetic operations were being created: crossover, position mutation, orientation mutation and permutation. In addition, the reproduction operation, which does not change the organisms, is implemented.

The one-point crossover operation for ensuring the exchanging of genetic material between organisms was used. The mutation of position or orientation randomly selects individual cassette in the production storage layout and randomly change its position or orientation. Changes of the orientation of an individual cassette can be made arbitrary or by predetermined angles (e.g. $0^{\circ}, 45^{\circ}, 90^{\circ}$ ). The permutation swaps the positions of randomly selected two individual cassettes, where the orientation of both is intact.

In the paper, the population size of 100 organisms was used. The maximum number of generations was 100 for all runs. For selection of organisms, the tournament method with the tournament size of 4 was used. The maximum number of generations was selected as the stopping criteria for the purposes of this paper. The following probability parameters were selected for the simulated evolution control: 0.4 for crossover, 0.2 for position mutation, 0.2 for orientation mutation, 0.1 for permutation and 0.1 for reproduction probability. 100 independent runs of the system were conducted. 


\section{THE RESULTS OF PATH PLANNING AND PRODUCTION STORAGE OPTIMIZATION SYSTEM}

Based on Fig. 6 storage 1 and 3 are the same. Accordingly, path planning and production storage optimization was conducted for storage 1 and 2, only.

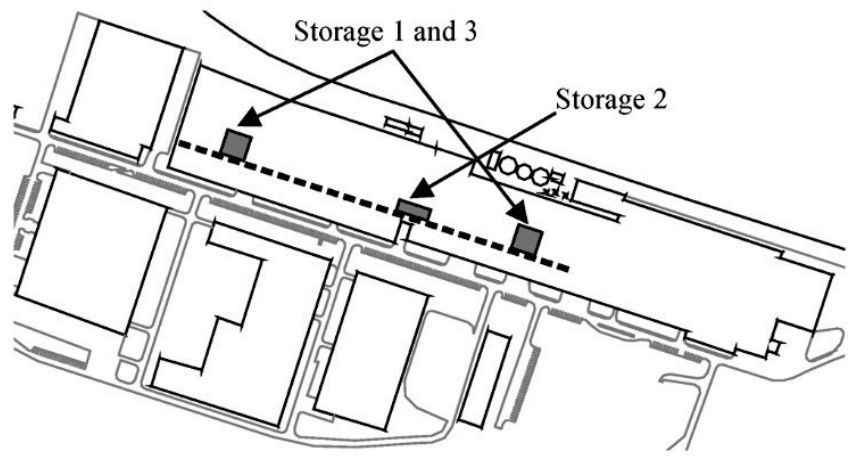

Figure 6 Storages 1, 2 and 3

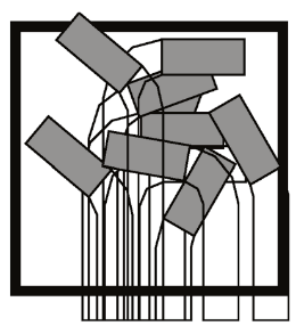

Figure 7 The best production storage layout in randomly generated population

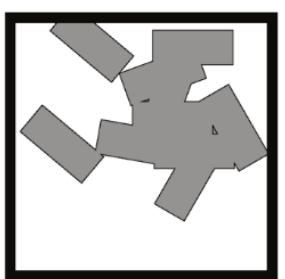

a)

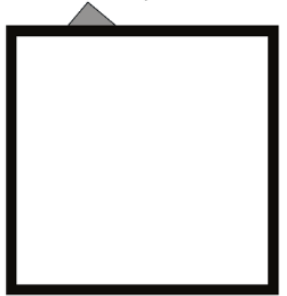

c)

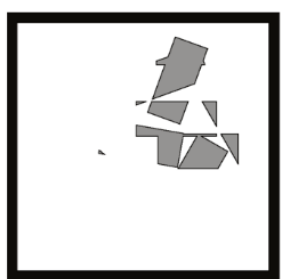

b)

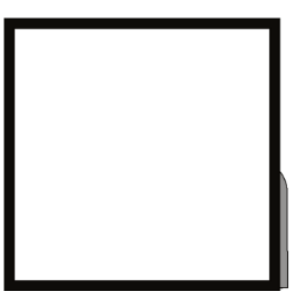

d)
Figure 8 Area of all cassettes in production storage (a), intersection area between cassettes (b), area of cassettes outside production storage (c) and area of electric transporter's paths outside production storage (d) of the best production storage layout in randomly generated population

The run of the system for storage 1 (and 2) started with random generation of population. The best production storage layout is presented in Fig. 7. The area of all cassettes in production storage $(A)$ is $168.24 \mathrm{~m}^{2}$, intersection area between cassettes $(A C)$ is $37.75 \mathrm{~m}^{2}$, area of cassettes outside production storage $(A c o s)$ is $1.34 \mathrm{~m}^{2}$ and area of electric transporter's paths outside production storage (Aetos) is
$20.92 \mathrm{~m}^{2}$ (Fig. 7). The evaluation function $F$ value for the best production storage layout is thus -431.86 .

Fig. 9 shows the worst production storage layout in randomly generated population with evaluation function $F$ value of $-4,435.76$.

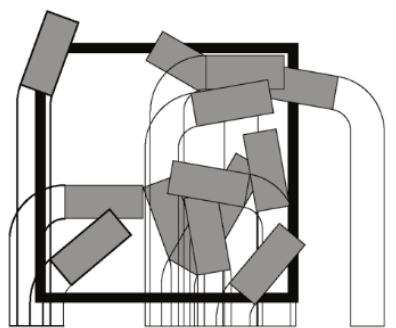

Figure 9 The worst production storage layout in randomly generated population

The best production storage layout was obtained in $93^{\text {rd }}$ generation (Fig. 10). Its evaluation function $F$ value is simply the area of all cassettes in production storage -252 .

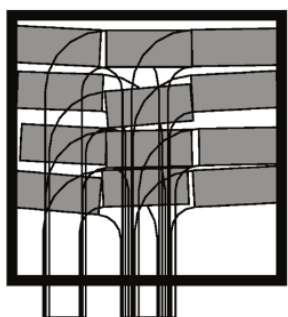

Figure 10 The best production storage layout obtained in 93rd generation

Similarly, the best production storage layout for storage 2 was obtained (Fig. 11). Its evaluation function $F$ value is 126.

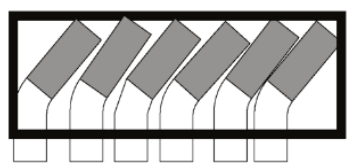

Figure 11 The best production storage layout for storage 2

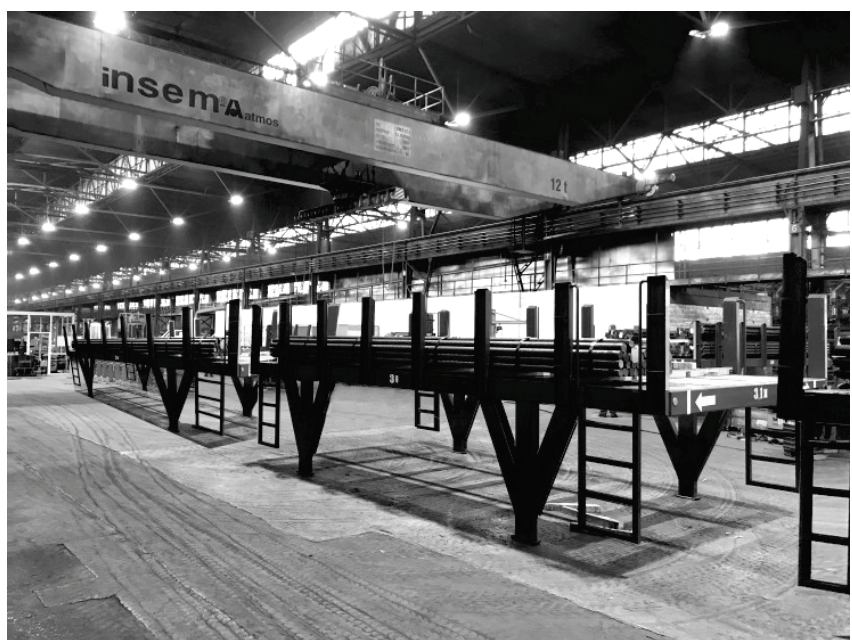

Figure 12 The actual production storage layout for storage 3 
The actual arrangement of the production storage 1 and 3 are actually the same like obtained using genetic algorithm. Fig. 12 shows the actual production storage layout for storage 3.

\section{CONCLUSIONS}

In this paper, the universal system using CAD geometry is presented. AutoCAD environment and in-house developed AutoLISP system were used. The developed system was used for path planning and production storage optimization (i.e. storage spaces for cassettes) in steel plant in Slovenia. For the optimization, the genetic algorithm was used as a search strategy. The organisms (i.e. production storage layouts) consist of genes (i.e. individual electric transporter's paths). The best organism in the population represents the best production storage layout. For changing organisms, the following genetic operations were used: crossover, position mutation, orientation mutation and permutation. The individual production storage layout was evaluated based on area of all cassettes in production storage, intersection area between cassettes, area of cassettes outside production storage area and area of electric transporter's paths outside production storage. Used evolutionary parameters were:

- the population size: 100 ,

- the maximum number of generations: 100 ,

- the tournament method with the tournament size of 4 ,

- crossover probability: 0.4 ,

- probability of position mutation: 0.2 ,

- probability of orientation mutation: 0.2 ,

- probability of permutation: 0 , and

- 0.1 for reproduction probability.

The production storage layout was obtained for two different storage locations. The number of cassettes in the first and in the second storage is 12 and 6, respectively. The obtained layouts were actually the same as they are used in the chosen steel plant in Slovenia. In the future the generation of path trajectories of electric transporter will be enhanced with additional complex manoeuvres (e.g. reverse driving, several different turning radiuses) and 3-dimensional objects.

\section{Notice}

The paper will be presented at MOTSP 2020 International Conference Management of Technology - Step to Sustainable Production, which will take place from $30^{\text {th }}$ September $-2^{\text {nd }}$ October 2020 in Bol, island Brač (Croatia). The paper will not be published anywhere else.

\section{REFERENCES}

[1] Rao, N. P. (1975). Two-Stage Production Systems with Intermediate Storage. AIIE Transactions, 7(4), 414-421. https://doi.org/10.1080/05695557508975025

[2] Martin, G. E. (1990). Optimal buffer storage capacity in unpaced lines. Computers \& Industrial Engineering, 18(3), 401-405. https://doi.org/10.1016/0360-8352(90)90062-Q

[3] Altiok, T. \& Stidham, S. (1983). The Allocation of Interstage
Buffer Capacities in Production Lines. IIE Transactions, 15(4), 292-299. https://doi.org/10.1080/05695558308974650

[4] Drira, A., Pierreval, H., \& Hajri-Gabouj, S. (2007). Facility layout problems: A survey. Annual Reviews in Control, 31(2), 255-267. https://doi.org/10.1016/j.arcontrol.2007.04.001

[5] Meller, R. D. \& Gau, K.-Y. (1996). The facility layout problem: Recent and emerging trends and perspectives. Journal of Manufacturing Systems, 15(5), 351-366. https://doi.org/10.1016/0278-6125(96)84198-7

[6] McKendall, A. R. \& Hakobyan, A. (2010). Heuristics for the dynamic facility layout problem with unequal-area departments. European Journal of Operational Research, 201(1), 171-182. https://doi.org/10.1016/j.ejor.2009.02.028

[7] Liang, L. Y. \& Chao, W. C. (2008). The strategies of tabu search technique for facility layout optimization. Automation in Construction, 17(6), 657-669. https://doi.org/10.1016/j.autcon.2008.01.001

[8] Azadeh, A., Moghaddam, M., Asadzadeh, S. M., \& Negahban, A. (2011). An integrated fuzzy simulation-fuzzy data envelopment analysis algorithm for job-shop layout optimization: The case of injection process with ambiguous dana. European Journal of Operational Research, 214(3), 768-779. https://doi.org/10.1016/j.ejor.2011.05.015

[9] Hani, Y., Amodeo, L., Yalaoui, F., \& Chen, H. (2007). Ant colony optimization for solving an industrial layout problem. European Journal of Operational Research, 183(2), 633-642. https://doi.org/10.1016/j.ejor.2006.10.032

[10] Önüt, S., Tuzkaya, U. R., \& Doğaç, B. (2008). A particle swarm optimization algorithm for the multiple-level warehouse layout design problem. Computers \& Industrial Engineering, 54(4), 783-799. https://doi.org/10.1016/j.cie.2007.10.012

[11] Kovačič, M., Rožej, U., \& Brezočnik, M. (2013). Genetic Algorithm Rolling Mill Layout Optimization. Materials and Manufacturing Processes, 28(7), 783-787. https://doi.org/10.1080/10426914.2012.718475

[12] Leu, M. C., ElMaraghy, H. A., Nee, A. Y. C., Ong, S. K., Lanzetta, M., Putz, M., Zhu, W., \& Bernard, A. (2013). CAD model based virtual assembly simulation, planning and training. CIRP Annals, 62(2), 799-822. https://doi.org/10.1016/j.cirp.2013.05.005

[13] Michalos, G., Spiliotopoulos, J., Makris, S., \& Chryssolouris, G. (2018). A method for planning human robot shared tasks. CIRP Journal of Manufacturing Science and Technology, 22, 76-90. https://doi.org/10.1016/j.cirpj.2018.05.003

[14] Zha, X. F. \& Du, H. (2001). Generation and Simulation of Robot Trajectories in a Virtual CAD-Based Off-Line Programming Environment. The International Journal of Advanced Manufacturing Technology, 17(8), 610-624. https://doi.org/10.1007/s001700170145

[15] Kovacic, M. \& Brezocnik, M. (2017). A Universal CAD System for Cutting Stock Problem. International Journal of Simulation Modelling, 16(2), 302-312. https://doi.org/10.2507/IJSIMM16(2)10.387

[16] Kovačič, M., Rožej, U., \& Brezočnik, M. (2013). Genetic algorithm rolling mill layout optimization. Materials and Manufacturing Processes, 28(7). https://doi.org/10.1080/10426914.2012.718475

[17] Jin, Y., He, Y., Fu, G., Zhang, A., \& Du, J. (2017). A nonretraction path planning approach for extrusion-based additive manufacturing. Robotics and Computer-Integrated Manufacturing, 48, 132-144. https://doi.org/10.1016/j.rcim.2017.03.008

[18] Kovacic, M. \& Balic, J. (2003). Evolutionary programming of a CNC cutting machine. International Journal of Advanced Manufacturing Technology, 22(1-2). 
https://doi.org/10.1007/s00170-002-1450-8

[19] Kovacic, M. \& Brezocnik, M. (2017). A universal cad system for cutting stock problem. International Journal of Simulation Modelling, 16(2). https://doi.org/10.2507//JSIMM16(2)10.387

[20] Goldberg, E. G. (1989). Genetic Algorithms in Search, Optimization and Machine Learning, $1^{\text {st }}$, Addison-Wesley Longman Publishing Co., Inc. Boston, MA, USA.

[21] Brezocnik, M. \& Kovacic, M. (2003). Integrated Genetic Programming and Genetic Algorithm Approach to Predict Surface Roughness. Materials and Manufacturing Processes, 18(3), 475-491. https://doi.org/10.1081/AMP-120022023

[22] Balic, J., Kovacic, M., \& Vaupotic, B. (2006). Intelligent programming of CNC turning operations using genetic algorithm. Journal of Intelligent Manufacturing, 17(3). https://doi.org/10.1007/s10845-005-0001-1

[23] Kovacic, M., Brezocnik, M., Pahole, I., Balic, J., \& Kecelj, B. (2005). Evolutionary programming of CNC machines. Journal of Materials Processing Technology, 164-165. https://doi.org/10.1016/j.jmatprotec.2005.02.047

[24] Kovačič, M. \& Šarler, B. (2011). Genetic algorithm-based batch filling scheduling in the steel industry. Materials and Manufacturing Processes, 26(3). https://doi.org/10.1080/10426914.2010.525576

[25] Stopar, K., Kovačič, M., Kitak, P., \& Pihler, J. (2017). Electric arc modeling of the EAF using differential evolution algorithm. Materials and Manufacturing Processes, 32(10), 1189-1200. https://doi.org/10.1080/10426914.2016.1257859

\section{Authors' contacts:}

Miha Kovačič, PhD, Assoc. Prof. (Corresponding author)

Štore Steel d.0.0.

Železarska cesta 3, 3220 Štore, Slovenia

Faculty of Mechanical Engineering, University of Ljubljana,

Aškerčeva cesta 6, 1000 Ljubljana, Slovenia

miha.kovacic@store-steel.si

Goran Đukić, PhD, Full Prof.

Fakultet strojarstva i brodogradnje, Sveučilište u Zagrebu, Ivana Lučića 5, 10002 Zagreb, Croatia

goran.dukic@fsb.hr

Brigita Gajšek, PhD, Assist. Prof.

Faculty of Logistics, University of Maribor,

Mariborska cesta 7, 3000 Celje, Slovenia

Klemen Stopar, PhD

Štore Steel d.o.o.

Železarska cesta 3, 3220 Štore, Slovenia 